\title{
Desenvolvimento gonadal de fêmeas de matrinxã, Brycon amazonicus, submetidas a restrição alimentar
}

\author{
Gonadal development of matrinxã, Brycon amazonicus, females submitted to feed restriction
}

\author{
Antonio Cleber da Silva Camargo ${ }^{\mathrm{I}}$ Sérgio Fonseca Zaiden ${ }^{\mathrm{II}}$ Elisabeth Criscuolo Urbinati ${ }^{\mathrm{II}}$
}

RESUMO

O presente estudo avaliou o efeito de ciclos de restrição alimentar e realimentação (2/3 dias), aplicados durante seis meses antes da desova, no desenvolvimento gonadal de matrinxã. Na ocasião da desova, fêmeas alimentadas diariamente e submetidas ao regime alimentar experimental, selecionadas para a indução hormonal, foram sacrificadas para retirada das gônadas e do fígado, com os quais se calculou o IGS (índice gonadossomático) e o IHS (índice hepatossomático), sendo os ovários processados para análise histológica. Não houve alteração no peso relativo dos ovários e fígado, e o desenvolvimento gonadal não foi afetado pelo esquema alimentar. Os valores de IGS foram de $5,09 \pm 4,98 \%$ e $9,79 \pm 4,17 \%$ e os de IHS foram de 0,84 $\pm 0,07 \%$ e $0,91 \pm 0,11 \%$, para as fêmeas controle e experimentais, respectivamente, sem diferenças significativas entre os grupos. Os ovários de peixes dos dois grupos apresentaram as mesmas características do estádio maduro, com predominância de ovócitos na fase final de maturação, repletos de vitelo. $O$ estudo indica que a restrição alimentar não afetou a preparação das fêmeas para a reprodução e que ciclos adequados de restrição e realimentação poderão ser aplicados na criação do matrinxã, assegurando menores custos de produção.

Palavras-chave: Matrinxã, desenvolvimento gonadal, ovários, restrição alimentar

\section{ABSTRACT}

The present study evaluated the effect of cycles of feed restriction and refeeding (2/3 days) during 6 months before the spawning on the gonadal development of matrinxã. At the spawning time, females selected to hormonal induction were killed for gonad and liver removal and GSI (gonadosomatic index) and HSI (hepatosomatic index) were calculated. Ovaries were processed for histological analysis. The feeding regime did not alter GSI and HSI and the gonadal development. The values of GSI were $5.09 \pm 4.98 \%$ and $9.79 \pm 4.17 \%$, and of $H S I$ were $0.84 \pm 0.07 \%$ and $0.91 \pm 0.11 \%$, in control and experimental females, respectively, without significant difference between groups. Ovaries from both fish groups showed the same charachteristics of the mature stage, with predominance of oocytes in the final phase of maturation, presenting large amount of yolk. The study indicates that the feed restriction did not affect the matrinxã ability to reproduction and that cycles of feed restriction and refeeding can be used in matrinxã farming leading to lower production costs.

Key words: Matrinxã, gonadal development, ovary, food restriction.

\section{INTRODUÇÃO}

Em peixes reofílicos, a redução da ingestão de alimentos ocorre naturalmente durante a migração para a desova (MACKENZIE et al., 1998). Entretanto, em sistema de criação, este acesso é facilitado pela oferta diária de alimento.

A redução de alimentos tem sido testada como estratégia para avaliar o desempenho dos peixes, mas os resultados são divergentes, provavelmente pelas diferenças de protocolo. Em alguns estudos com peixes, a restrição afetou a maturação nos machos (ROWE \& THORPE, 1990; REIMERS et al., 1993) e em outros não (JOBLING et al., 1993; TOWNSHEND \& WOOTTON, 1984). Os efeitos negativos da privação de alimento são mais intensos em fêmeas (SILVERSTEIN \& SHIMA, 1994; KARLSEN et al., 1995; DUSTON \& SAUNDERS, 1999), dependendo da

\footnotetext{
INúcleo de Ciência Agrárias, Universidade Federal de Minas Gerais (UFMG), Montes Claros, MG, Brasil.

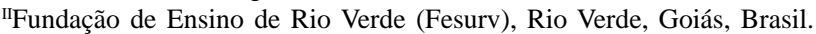

IIIDepartamento de Morfologia e Fisiologia Animal, Faculdade de Ciências Agrárias e Veterinárias (FCAV), Universidade Estadual Paulista (UNESP), Jaboticabal, SP, Brasil. Email: bethurb@caunesp.unesp.br. Autor para correspondência.
} 
duração da restrição. Restrição iniciada 40 dias antes da desova não afetou a capacidade reprodutiva de trutas arco-íris (RIDELMAN et al., 1984), mas uma restrição mais prolongada reduziu a fecundidade na mesma espécie (BAGENAL, 1969; KNOX et al., 1988) e em Cichlasoma nigrofasciatum (TOWNSHEND \& WOOTTON, 1984). Entretanto, nenhum prejuízo no desenvolvimento ovariano foi relatado em Macquaria ambigua após redução da alimentação (COLLINS \& ANDERSON, 1999).

Entre as várias espécies onívoras com grande potencial para criação na América do Sul (SAINT-PAUL, 1986), destaca-se o matrinxã, Brycon amazonicus (SPIX \& AGASSIZ, 1829). Este estudo analisou o desenvolvimento gonadal de fêmeas do matrinxã submetidas a ciclos de restrição alimentar (dois dias) e realimentação (três dias), por seis meses antes da desova.

\section{MATERIAL E MÉTODOS}

\section{Animais e manejo}

Um lote de 120 reprodutores (3 anos, peso médio de 1,65kg) distribuído em oito tanques externos, de $40 \mathrm{~m}^{2}$, com fluxo constante de água, foi observado por 15 dias para quantificação da ração oferecida. Os tanques foram sorteados para distribuição dos tratamentos, controle e experimental (quatro tanques/ tratamento, 15 peixes/tanque). Os peixes-controle receberam ração diariamente (uma vez ao dia), a $2 \%$ da biomassa, fixada no início do experimento, e os peixes do grupo experimental foram submetidos a ciclos de três dias de alimentação seguidos de dois dias de restrição durante os seis meses anteriores à desova (abril a outubro). Quando alimentados, os peixes do grupo experimental recebiam a mesma quantidade oferecida aos peixes controle. Foi utilizada uma ração comercial extrusada (28\% de proteína bruta).

\section{Coleta e processamento do material biológico}

Por ocasião da desova das fêmeas, de ambos, os grupos selecionadas para indução hormonal, 10 peixes foram sacrificados para retirada dos ovários e fígado. Os órgãos foram pesados para cálculo do IGS (índice gonadossomático) e do IHS (índice hepatossomático), respectivamente, segundo a fórmula: \{(peso do órgão/peso corporal)x100\}. Os ovários foram fixados em Bouin por 24 horas, lavados em água corrente para retirada do excesso do fixador, lavados em álcool 70\% e hidróxido de amônio $(0,5 \mathrm{~mL}$ $\left.100 \mathrm{~mL}^{-1}\right)$, desidratados em série crescente de álcool etílico, diafanizadas em xilol e incluídos em parafina para obtenção de cortes que foram corados em hematoxilina-eosina, analisados e fotografados em microscópio Axioskop da Zeiss, modelo MC80DX.
Os dados de IGS e IHS foram submetidos à análise de variância e as médias comparadas pelo teste de Tukey, utilizando-se o software SAS/STAT (1990). Os resultados da análise histológica foram apenas descritivos.

\section{RESULTADOS}

Diariamente foram aferidos a temperatura da água dos viveiros $\left(24,69 \pm 0,20^{\circ} \mathrm{C}\right)$, a concentração de oxigênio dissolvido $\left(7,4 \pm 0,6 \mathrm{mg} \mathrm{L}^{-1}\right)$ e o $\mathrm{pH}(7,4 \pm 0,4)$, e, a cada três dias, determinou-se a concentração de amônia total $(0,01 \mathrm{mg} / \mathrm{L})$ e a alcalinidade $\left(11,8 \pm 1,9 \mathrm{mg} \mathrm{L}^{-1}\right)$. Os valores encontrados estão dentro da faixa recomendada para cultivo de espécies tropicais (BOYD, 1990) e não apresentaram alterações que pudessem interferir no desenvolvimento gonadal.

Índice gonadossomático (IGS) e índice hepatossomático (IHS)

Os valores de IGS foram de 5,09 $44,98 \%$ e $9,79 \pm 4,17 \%$ e os de IHS foram de $0,84 \pm 0,07 \%$ e $0,91 \pm 0,11 \%$, para as fêmeas-controle e as experimentais, respectivamente, sem que tenham sido verificadas diferenças significativas entre os grupos.

Descrição histológica dos ovários nos grupos controle e experimental

O regime alimentar não afetou o desenvolvimento gonadal. Os ovários apresentaram as mesmas características do estádio de maturação final nas fêmeas dos dois grupos. Verificou-se a ocorrência de lamelas ovulígeras com túnica albugínea bastante delgada e predominância de ovócitos na fase de maturação final. Havia pequena quantidade de ovócitos na fase cromatina-nucléolo e perinucleolar ocupando os interstícios entre os ovócitos em maturação final, que se caracterizaram por serem as maiores células da linhagem germinativa encontradas, apresentando núcleo central acidófilo, de contorno irregular, delimitado por grânulos de vitelo e presença de nucléolos basófilos em sua região periférica. $\mathrm{O}$ citoplasma estava tomado por grânulos de vitelo de tamanhos diversos e alvéolos corticais periféricos. A zona pelúcida era nítida, com típicas estrias radiais. A camada folicular (granulosa) e a teca eram bemdefinidas, formadas por células achatadas com núcleos também achatados (Figuras 1A, 1B). Verificou-se a presença de micrópila com parede da zona pelúcida mais espessa, apresentando célula micropilar cuneiforme (Figura 2B).

Os ovócitos na fase cromatina-nucléolo se apresentaram em ninhos com números variados de 


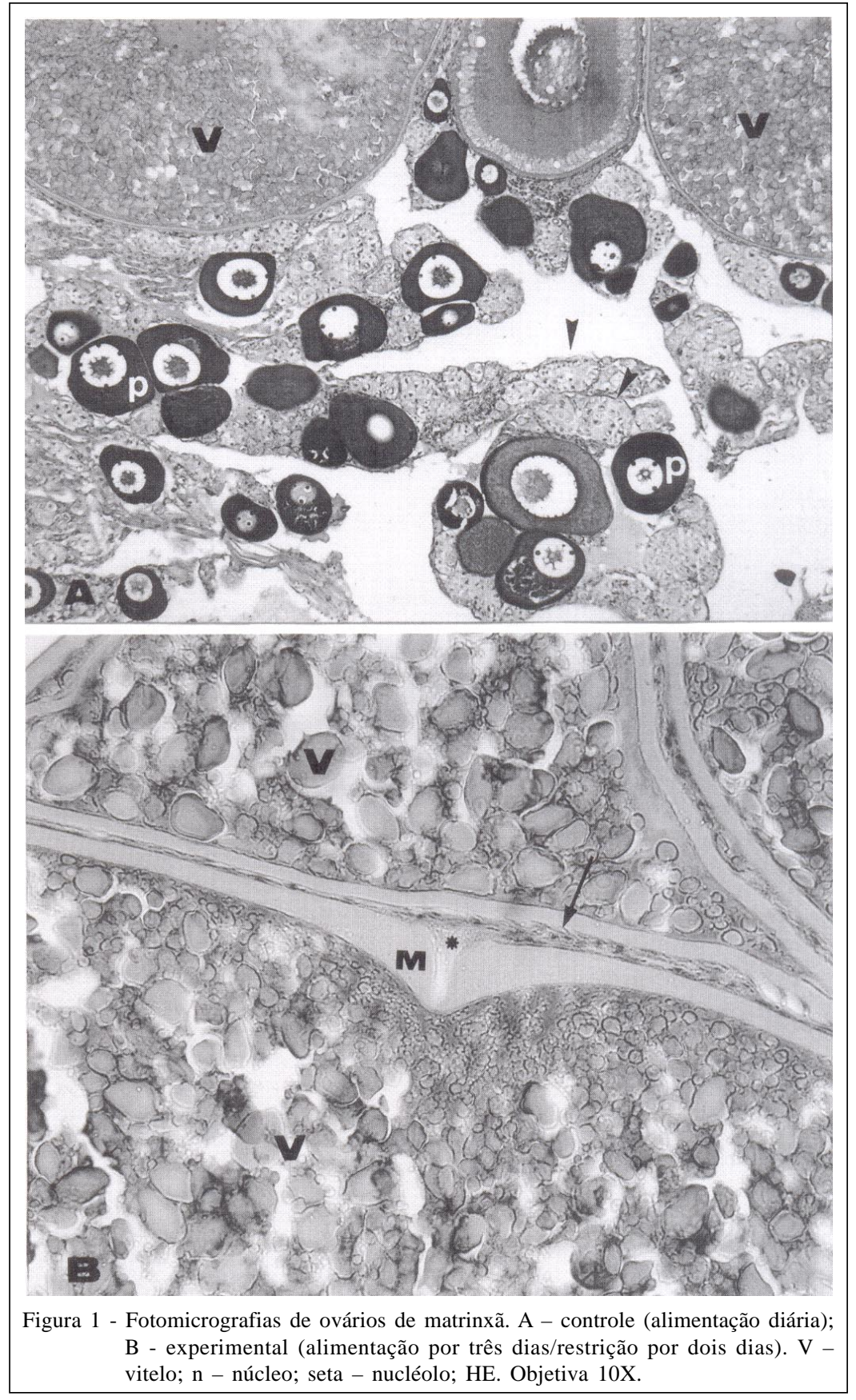

células nas fases iniciais da prófase I da meiose I, enquanto que os ovócitos perinucleolares se mostraram de tamanhos variados, com citoplasma bastante basófilo e núcleo redondo repleto de nucléolos também redondos em suas bordas (Figura 2A).

\section{DISCUSSÃO}

As fêmeas sob restrição alimentar responderam do mesmo modo que as alimentadas diariamente tanto em relação ao peso relativo dos ovários (IGS) e do fígado (IHS) como em relação ao desenvolvimento celular do ovário. O desenvolvimento gonadal foi classificado segundo ZANIBONI-FILHO \& RESENDE (1988), sendo identificados cinco estádios de acordo com os tipos celulares: estádio A (imaturo), estádio B (em maturação), estádio C (maduro), estádio $\mathrm{D}$ (esvaziado) e estádio R (repouso).

Os efeitos da redução de alimento na reprodução divergem em diferentes estudos, 

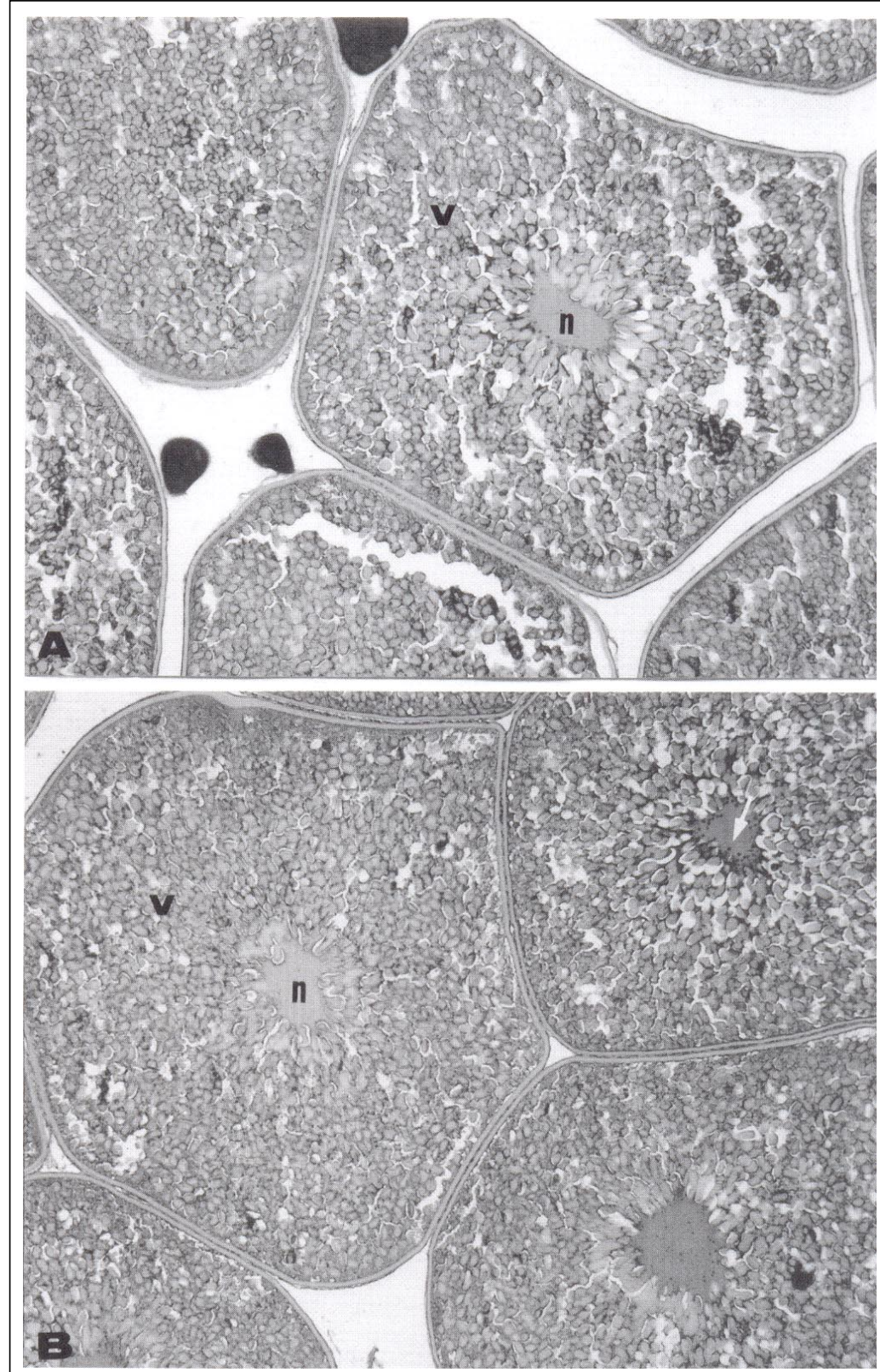

Figura 2 - Fotomicrografias de ovários de matrinxã. A - controle (alimentação diária); V - vitelo; p - ovócito no estágio perinucleolar; cabeça de seta - ninhos de ovócitos no estágio cromatina nucléolo. HE. Objetiva 10X. B - experimental (alimentação por três dias/restrição por dois dias). M - micrópila; * - célula micropilar; V - vitelo; seta - camada folicular. HE. Objetiva 40X.

dependendo de condições como espécie, sexo, tempo e severidade da redução, época do ano e fase do ciclo reprodutivo. Restrição em semanas alternadas, por seis meses, não afetou o número de machos maduros de Salvelinus alpinus (JOBLING et al., 1993), enquanto machos de Cichlasoma nigrofasciatum alimentados três vezes por semana, por quatro meses, não apresentaram desenvolvimento testicular alterado
(TOWNSHEND \& WOOTTON, 1984). Por outro lado, houve redução de machos maduros de salmão do Atlântico quando a restrição alimentar ocorreu no inverno (REIMERS et al., 1993) e na primavera (ROWE \& THORPE, 1990).

Há evidências de que a restrição alimentar afeta mais as fêmeas (SILVERSTEIN \& SHIMA, 1994; KARLSEN et al., 1995; DUSTON \& SAUNDERS, 1999), 
dependendo da duração da restrição. Em truta arcoíris, não houve alteração no tamanho das gônadas, composição e viabilidade dos ovos quando a restrição começou 40 dias antes da desova (RIDELMAN et al., 1984), mas a restrição de nove meses diminuiu a fecundidade e resultou em ovos maiores (BAGENAL, 1969). A fecundidade também foi menor em Cichlasoma nigrofasciatum, alimentada três vezes por semana, por quatro meses, além de ocorrer aumento dos intervalos entre desovas e produção de ovos maiores (TOWNSHEND \& WOOTTON, 1984). Diferentemente, a redução diária de alimento pela metade, durante um ano, produziu ovos menores na truta arco-íris (KNOX et al., 1988), enquanto Clepea harengus submetidos à alimentação subótima apresentaram maior atresia ovariana e mudanças nos índices gonadossomático e hepatossomático (MA et al., 1998). Entretanto, a redução gradativa da oferta de ração, por 17 meses, para a Tilapia zillii, não afetou o tamanho dos ovócitos e a freqüência da desova, embora houvesse maior número de ovócitos pré-vitelogênicos e em início de vitelogênese (COWARD \& BROMAGE, 1999).

No presente estudo, a restrição alimentar por seis meses não afetou o desenvolvimento dos ovários, que possuíam o mesmo peso relativo (IGS) e predominância de ovócitos em fase final de maturação, repletos de grânulos de vitelo. Estudo anterior (CARVALHO, 2001) com a mesma espécie, criada por um ano no mesmo regime alimentar deste estudo, destacou a capacidade metabólica e hormonal compensatória do matrinxã. Os peixes restritos (machos e fêmeas) tiveram crescimento corporal, índices metabólicos e níveis plasmáticos de testosterona similares aos dos peixes alimentados diariamente, sem alteração do número de indivíduos maduros.

Segundo BURTON (1994), o estado nutricional do peixe é mais importante que a quantidade de alimento oferecida durante a fase de transformação de ovócitos perinucleolares a vitelogênicos, o que sugere que a quantidade de alimento oferecida ao matrinxã foi suficiente para atender sua necessidade energética de manutenção e construção do ovário maduro. De acordo com MOMMSEN \& PLISETSKAYA (1991), a secreção de insulina estimulada pela presença de nutrientes no sangue é um mecanismo ativado na realimentação para seu aproveitamento como reserva energética. Além disso, sob privação de alimento, o peixe está preparado bioquimicamente para uma utilização mais eficiente do alimento disponível (CHRISTIANSEN \& KLUNGSOYER, 1987).

A restrição de alimento também não afetou o desenvolvimento ovariano de Macquaria ambigua, espécie de água doce da Austrália, cujo ambiente natural sofre alterações sazonais que afetam a oferta de alimentos (COLLINS \& ANDERSON, 1999), como ocorre com o matrinxã, o que pode ser um fator determinante da capacidade intrínseca de recuperação observada nestas espécies durante a disponibilidade de alimentos, após redução de sua oferta.

O processo de maturação gonadal nos peixes é bastante elaborado, com alta exigência de energia. Entretanto, a redução da ingestão de alimentos é um evento natural no ciclo biológico dos peixes, como na migração para a desova (MACKENZIE et al., 1998), de modo que a oferta diária de alimento na criação intensiva parece desnecessária.

\section{CONCLUSÕES}

O estudo mostra que a restrição alimentar, da forma e duração em que foi imposta (dois dias de restrição seguidos de três dias de realimentação por seis meses antes da desova), não afeta a capacidade das fêmeas de matrinxã de preparação para a reprodução. Ciclos adequados de restrição alimentar e realimentação poderão ser aplicados na criação do matrinxã e assegurar economia de ração, sem prejudicar sua capacidade homeostática.

\section{REFERÊNCIAS}

BAGENAL, T.B. The relationship between food supply and fecundity in brown trout Salmo truta L. Journal of Fish Biology, v.1, p.167-182, 1969.

BOYD, C.E. Water quality in warm water fish pond. Alabama: Auburn University. 1990. 482p.

BURTON, M.P.M. Critical period for nutritional control of early gametogenesis in female flounder, Pleuronectes americanus (Pisces: Teleostei). Journal of Zoology, v.233, p.405-415, 1994.

CARVALHO, E.G. Restrição alimentar e reprodução em peixes. 2001. 120f. Tese (Doutorado em Zootecnia) - Curso de Pós-graduação em Zootecnia, Universidade Estadual Paulista, UNESP, Jaboticabal, São Paulo.

COLLINS, A.L.; ANDERSON, T.A. The role of food availability in regulation reproductive development in female golden perch. Journal of Fish Biology, v.55, p.94-104, 1999.

COWARD, K.; BROMAGE, N.R. Spawning frequency, fecundity, egg size and ovarian histology in groups of Tilapia zillii maintained upon two distinct food ration sizes from first-feeding to sexual maturity. Aquatic Living Resources, v.12, n.1, p.11-22, 1999.

CHRISTIANSEN, D.C.; KLUNGSOYR, L. Metabolic utilization of nutrients and the effects of insulin in fish. Comparative Biochemistry and Physiology, v.88B, p.701-711, 1987.

DUSTON, J.; SAUNDERS, R.L. Effect of winter food deprivation on growth and sexual maturity of Atlantic salmon 
(Salmo salar) in seawater. Canadian Journal of Fisheries and Aquatic Sciences, v.56, p.201-207, 1999.

JOBLING, M. et al. The influence of previous feeding regime on the compensatory growth response of maturation and immature arctic charr, Salvelinus alpinus. Journal of Fish Biology, v.43, p.409-419, 1993.

KARLSEN, Ø. et al. Effects of periodic starvation on reproductive investment in first-time spawning Atlantic cod (Gadus morhua L). Aquaculture, v.133, p.159-170, 1995.

KNOX, D. et al. The effect of broodstock ration size on the composition of rainbow trout eggs (Salmo gairdneri). Aquaculture, v.69, p.93-104, 1988.

MA, Y. et al. Effects of ration on the maturation and fecundity in captive Atlantic herring (Clepea harengus). Canadian Journal of Fisheries and Aquatic Sciences, v.55, p.900908, 1998.

MACKENZIE, D.S. et al. Nutrient regulation of endocrine function in fish. Aquaculture, v.161, p.3-25, 1998.

MOMMSEN, T.P.; PLISETSKAYA, E.M. Insulin in fishes and agnathans: history, structure and metabolic regulation. Reviews of Aquatic Sciences, v.4, p.225-259, 1991.

REIMERS, E. et al. Compensatory growth and reduced maturation in second sea winter farmed Atlantic salmon following starvation in February and March. Journal of Fish Biology, v.43, p.805-810, 1993.

RIDELMAN, J.M. et al. The effect of short-term starvation on ovarian development and egg viability in rainbow trout (Salmo gairdneri). Aquaculture, v.37, p.133-140, 1984.

ROWE, D.K.; THORPE, J.E. Suppression of maturation in male Atlantic salmon (Salmo salar L.) parr by reduction in feeding and growth during spring months. Aquaculture, v.86, p.291-313, 1990.

SAINT PAUL, U. Potential for aquaculture of South America: the review. Aquaculture, v.54, p.205-240, 1986.

SAS/STAT User's Guide. Version 6. Fourth Edition, V.1, SAS Institute. Cary, NC, 1990. p.943.

SILVERSTEIN, J.T.; SHIMMA, H. Effect of restricted feeding on early maturation in female and male amago salmon (Oncorhynchus masou ishikawae). Journal of Fish Biology, v.45, p.1133-1135, 1994.

TOWNSHEND, T.; WOOTTON, R. Effects of food supply on reproduction of the convict ciclid, Cichlasoma nigrofasciatum. Journal of Fish Biology, v.24, p.91-104, 1984.

ZANIBONI-FILHO, E.; RESENDE, E.K. Anatomia de gônadas, escala de maturidade e tipo de desova do matrinxã, Brycon cephalus (Günther, 1869) (Teleostei: Characidae). Revista Brasileira de Biologia, v.48, n.4, p.833-844, 1988. 